\title{
New Interference Experiment with Asymmetric Double Slits by 1.2 MV FE-TEM
}

\author{
K. Harada ${ }^{1,3}$, T. Akashi ${ }^{2,4}$, K. Niitsu ${ }^{1}$, K. Shimada ${ }^{1}$, Y. A. Ono ${ }^{1}$, D. Shindo ${ }^{1,5}$, H. Shinada ${ }^{2}$ and S. Mori ${ }^{3}$ \\ 1. CEMS, RIKEN, Hatoyama, Saitama, Japan. \\ 2. RDG, Hitachi, Ltd., Hatoyama, Saitama, Japan. \\ 3. Materials Science, Osaka Prefecture Univ., Sakai, Osaka, Japan. \\ 4. Applied Quantum Physics, Kyushu Univ., Fukuoka, Fukuoka, Japan. \\ 5. IMRAM, Tohoku Univ., Sendai, Miyagi, Japan.
}

Wave-particle duality is one of the fundamental properties of fine particles pointed out by Feynman as one of the mysteries of quantum physics. Various experimental observations, for example by Tonomura et al. [1], have been reported on the wave-particle duality of electrons; however, this mysterious "whichway" issue has not been fully resolved yet. Recent technological developments have made it possible to perform double-slit (DS) experiments more easily and precisely. We used the following technologies and methods [2]: (1) highly coherent electron beams from a 1.2-MV field-emission transmission electron microscope (FE-TEM) [3], (2) asymmetric double slits (ADS) having slit widths that can be varied by using a biprism, (3) measurements under a "pre-Fraunhofer" condition at defocus distances $(\Delta f)$ shorter than those in the Fraunhofer condition, and (4) a direct electron detection camera system.

Figure 1 shows a schematic diagram of the optical system for the ADS experiment. The DS and two electron biprisms (I and II) are placed at the image planes, i.e. conjugate planes for the object plane. The biprism I was utilized to control the opening width of one of the slits to be asymmetric. The biprism II was used to shield the beam going through one of the single slits for the alternation between single-slit and double-slit experiments.

Figure 2 shows infocus image and pre-Fraunhofer patterns of the ADS recorded by the direct detection camera system in the electron counting mode. The rectangle openings of the ADS are of $0.12 \mu \mathrm{m}$ width for the left slit and $0.035 \mu \mathrm{m}$ width for the right slit, with a $0.8 \mu \mathrm{m}$ spacing between the slits.

Figure 3 shows a composite image of the categorized electrons in three colors: blue corresponds to electrons passed through only the left slit; green corresponds to electrons passed through only the right slit; and red corresponds to electrons passed through both slits simultaneously. The red electrons form a two-wave interference fringe pattern. Averaged intensity profile is shown above the image. An enlarged image pattern is shown in the inset.

In conclusion, we succeeded in categorizing electrons in three types in terms of the intensity profiles, although we could not completely perform the which-way experiment yet. We hope this trial will contribute to resolve the "which-way" issues [4].

\section{References:}

[1] A. Tonomura et al., Am. J. Phys. 57 (1989), p. 117. doi: 101119/1.16104.

[2] K. Harada et al., Scientific Reports 8 (2018), p. 1008. doi: 10.1038/s41598-018-19380-4.

[3] T. Akashi et al., Appl. Phys. Lett. 106 (2015), p. 074101. doi: 10.1063/1.4908175. 
[4] The authors would like to thank Prof. Takuya Hirano of Gakushuin University and Dr. Masato Tomita of the National Institute for Physiological Sciences for their stimulating discussions.

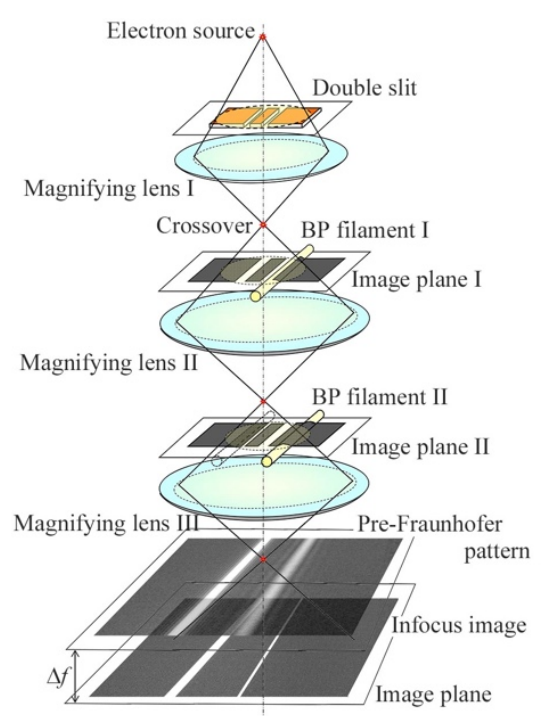

Figure 1. Schematic diagram of optical system.

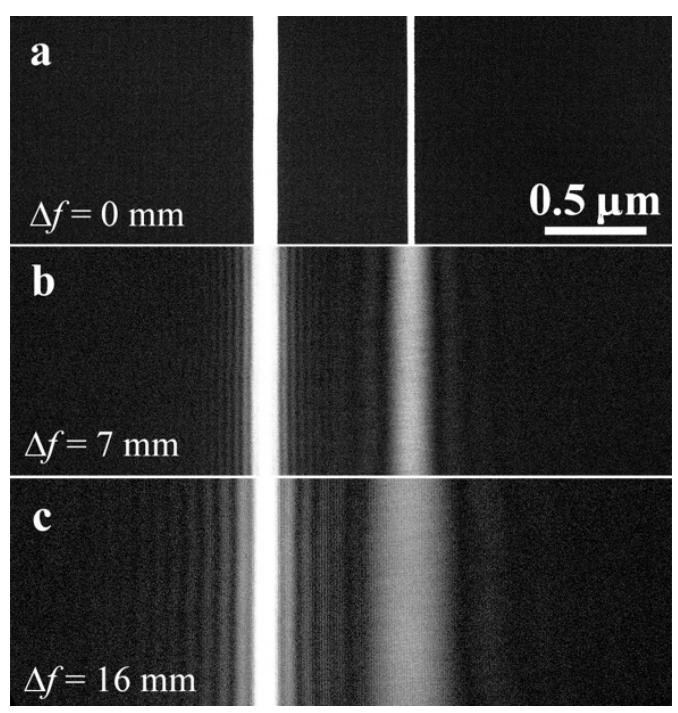

Figure 2. Pre-Fraunhofer patterns of an ADS.

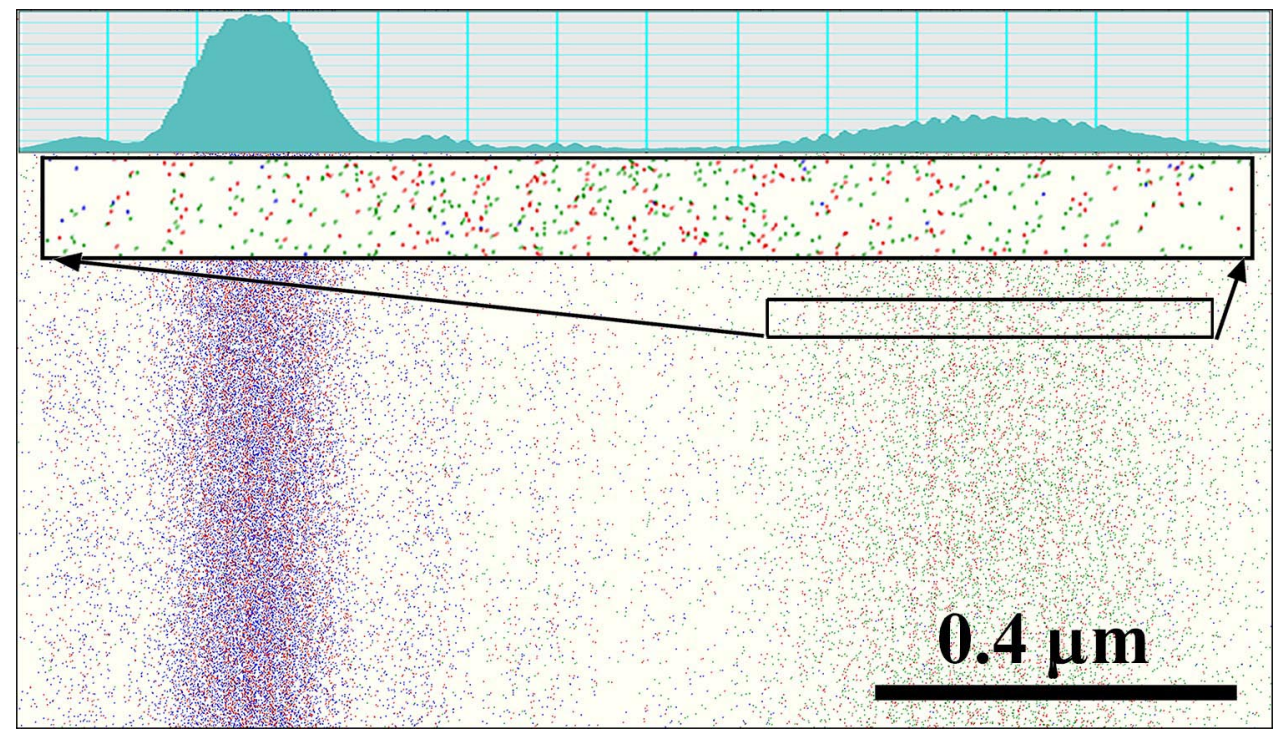

Figure 3. Composite image of color-coded electrons of the present version of the "which-way" doubleslit experiment: blue colored electrons passed through only the left slit; red colored electrons passed though both slits simultaneously; green colored electrons passed through only the right slit. Upper part is an averaged intensity profile of the pre-Fraunhofer fringe pattern. The inset indicates an enlarged image of the data in the rectangle area shown below. 